\title{
Viscosity Solution of Mean-Variance Portfolio Selection of a Jump Markov Process with No-Shorting Constraints
}

\author{
Moussa Kounta \\ The College of the Bahamas, School of Mathematics, Physics and Technology, P.O. Box 4912, Nassau, Bahamas \\ Correspondence should be addressed to Moussa Kounta; moussakounta@gmail.com
}

Received 30 December 2015; Accepted 15 March 2016

Academic Editor: Jinde Cao

Copyright (C) 2016 Moussa Kounta. This is an open access article distributed under the Creative Commons Attribution License, which permits unrestricted use, distribution, and reproduction in any medium, provided the original work is properly cited.

\begin{abstract}
We consider the so-called mean-variance portfolio selection problem in continuous time under the constraint that the shortselling of stocks is prohibited where all the market coefficients are random processes. In this situation the Hamilton-Jacobi-Bellman (HJB) equation of the value function of the auxiliary problem becomes a coupled system of backward stochastic partial differential equation. In fact, the value function $V$ often does not have the smoothness properties needed to interpret it as a solution to the dynamic programming partial differential equation in the usual (classical) sense; however, in such cases $V$ can be interpreted as a viscosity solution. Here we show the unicity of the viscosity solution and we see that the optimal and the value functions are piecewise linear functions based on some Riccati differential equations. In particular we solve the open problem posed by Li and Zhou and Zhou and Yin.
\end{abstract}

\section{Introduction}

The mean-variance approach proposed in 1952 by the Nobel prize winning economist Markowitz [1] has become the foundation of modern finance by discovering the static meanvariance portfolio selection formulation in a market in which shorting is not allowed. This theory has inspired numerous extensions and applications. For instance, $\mathrm{Li}$ and $\mathrm{Ng}$ [2] and Zhou and Li [3] successfully extended the unconstrained mean-variance portfolio selection formulation to the multiperiod setting. Zhou and Yin [4] consider the mean-variance portfolio selection problem in continuous time where the market parameters including the bank interest rate and the appreciation and volatility rates of the stocks depend on the market mode that switches among a finite number of states where random regime switching is assumed to be independent of the underlying Brownian motion. This essentially renders the underlying market incomplete. A Markov chain modulated diffusion formulation is employed to model the problem and Zhou and Yin [4] use the techniques of stochastic linear quadratic (LQ) control to derive mean-variance efficient portfolios and efficient frontier based on solutions of two systems of linear ordinary differential equations.
After $\mathrm{Li}$ and $\mathrm{Ng}$ published [2], Markowitz suggested that one of them extends the results to the dynamic meanvariance formulation with no-shorting constraint and proposed a conjecture of a piecewise quadratic value function for such a situation. Influenced by Markowitz's comments, Li et al. [5] formulated the LQ control problem by constraining the control portfolio to take nonnegative values due to the no-shorting restriction on the market mode (not random processes). They derived the optimal portfolio policy for the continuous-time mean-variance model with no-shorting constraint using the duality method [6].

However, there are several interesting problems that deserve further investigation; for instance, $\mathrm{Li}$ et al. [5] open a problem by stating in their conclusion that "an immediate open problem is to extend the results in this paper to the case where all the market coefficients are random processes." In this paper we solve this problem.

By making use of the techniques of LQ control, we see that, in an attempt to pursue the method of dynamic programming in the auxiliary problem, the value function which is a generalized solution to the Hamilton-Jacobi equation coupled is not smooth enough to satisfy the dynamic programming equations in the classical or usual sense. 
A difficulty with the concept of generalized solution is that the dynamic programming together with the boundary data typically has many generalized solutions. Among them, there is one provided by Crandall and Lions [7], called the viscosity solution, which is the natural generalized solution. This unique viscosity solution turns out to coincide with the value function $V$ [8]. The central component of our solution to the problem of $\mathrm{Li}$ et al. [5] is the proof of the unicity of the viscosity solution of the value function of the auxiliary problem, which we establish by adapting the techniques of [9]. By making use of the duality method, we also derive a solution for efficient portfolio. The value function of the auxiliary problem depends on a set of Riccati differential equations and we use the Magnus approach to provide the solution. A work is in progress to develop numerical implementation. This will be subject of a future publication.

\section{Viscosity Solutions for Weakly Coupled Systems of Second-Order Hamilton-Jacobi- Bellman Equation}

2.1. Notation. We make use of the following notations:

(i) $(\Omega, \mathscr{F}, P)$ : a fixed probability space on which we defined standard $n$-dimensional Brownian motion $W(t) \equiv\left(W_{1}(t), \ldots, W_{n}(t)\right)^{\prime}$ and continuous-time stationary Markov chain $\alpha(t)$ taking value in a finite state space $\mathscr{M}=\{1,2, \ldots, m\}$ such that $W(t)$ and $\alpha(t)$ are independent of each other. The Markov chain has a generator $Q=\left(q_{i j}\right)_{m \times m}$ and stationary transition probabilities:

$p_{i j}(t)=p(\alpha(t)=j \mid \alpha(0)=i)$,

$$
t \geq 0, i, j=1,2, \ldots, m \text {. }
$$

(ii) Define $\mathscr{F}_{t}=\sigma\{(W(s), \alpha(s)): 0 \leq s \leq t\}$.

(iii) $\mathscr{B}(\Sigma)=\sigma$-algebra of Borel sets of $\Sigma$.

(iv) Consider the following:

$$
\begin{aligned}
& E_{t x} \Phi(x(s))=\int_{\Sigma} \Phi(y) \widehat{P}(t, x, s, d y), \quad t<s . \\
& \widehat{P}(t, x, s, B)=P(x(s) \in B \mid x(t)=x),
\end{aligned}
$$

$$
\forall B \in \mathscr{B}(\Sigma)
$$

(v) Hilbert space $\mathscr{H}$ with the norm $\|\cdot\|_{\mathscr{H}}$ : define the Banach space

$L_{\mathscr{F}}^{2}(0, T ; \mathscr{H})=\left\{\varphi(\cdot) \mid \varphi(\cdot)\right.$ is an $\mathscr{F}_{t}$-adapted,

$\mathscr{H}$-valued measurable process on $[a, b]$,

$$
\left.E \int_{a}^{b}\|\varphi(t, \omega)\|_{\mathscr{H}} d t<\infty\right\},
$$

with norm

$$
\|\varphi(\cdot)\|_{\mathscr{F}, 2}=E \int_{a}^{b}\|\varphi(t, \omega)\|_{\mathscr{H}} d t<\infty
$$

(vi) $M^{\prime}$ : the transpose of any vector or matrix.

(vii) $M_{j}$ : the $j$ th component of any vector $M$; we will use indifferently this notation $M(t, x, u, i) \equiv$ $M_{i}(t, x, u)$.

(viii) $C([0, T] ; X)$ : the Banach space of $X$-valued continuous functions on $[0, T]$.

(ix) $C^{2}\left([0, T] \times \mathbb{R}^{n}\right)$ : the space of all twice continuously differentiable functions on $[0, T] \times \mathbb{R}^{n}$.

(x) Consider $D_{x}=\partial(\cdot) / \partial x, D_{x}^{2}=\partial^{2}(\cdot) / \partial x^{2}, D^{\alpha}(\cdot)=$ $\partial^{|\alpha|}(\cdot) / \partial x_{1}^{\alpha_{1}} \cdots \partial x_{n}^{\alpha_{n}}$, and $\dot{f}(t)=(d / d t) f(t), \partial_{t}=\partial / \partial t$.

(xi) Consider

$$
\mathcal{Q}=[0, T] \times \mathscr{D}, \quad \mathscr{D} \subset \mathbb{R} .
$$

(xii) Kronecker delta symbol:

$$
\delta_{i j}(t)= \begin{cases}0 & \text { if } i \neq j \\ 1 & \text { if } i=j .\end{cases}
$$

(xiii) $[A, B] \equiv A B-B A$ (Lie bracket), $A, B$ matrices with appropriate dimension.

(xiv) Consider $W^{k, p}(\Omega)=\left\{u \in L^{p}(\Omega): D^{\alpha} u \in L^{p}(\Omega), \quad 1 \leq\right.$ $p \leq \infty, \forall|\alpha| \leq k\}$

(xv) $C_{b}(\Sigma)$ is bounded function in $\Sigma$.

(xvi) If $F$ is a real-valued function on a set $U$ which has a minimum on $U$, then

$$
\underset{v \in U}{\operatorname{argmin}} F(v)=\left\{v^{*} \in U: F\left(v^{*}\right) \leq F(v), \forall v \in U\right\} .
$$

\section{Notion of Viscosity Solution}

We consider the following coupled system of backward PDEs:

$$
\begin{aligned}
& \partial_{t} V_{i}(t, x) \\
& +\inf _{u \geq 0}\left\{\frac{1}{2} \bar{g}_{i}(t, x(t), u) \bar{g}_{i}(t, x(t), u)^{\prime} D_{x}^{2} V_{i}(t, x)\right. \\
& \left.+\bar{f}_{i}(t, x(t), u) D_{x} V_{i}(t, x)\right\}-\sum_{j \neq i} q_{i j}\left[V_{i}(t, x)\right. \\
& \left.-V_{j}(t, x)\right]=0, \\
& V_{i}(T, x)=g(x) \\
& \text { and the conditions on matrix }\left(q_{i j}\right)_{1 \leq i, j \leq n} \text { are } \\
& \quad q_{k l}>0, \quad \text { for } k \neq l, q_{k k}<0, \\
& \sum_{l=1}^{m} q_{k l}=0 \quad \text { for } k=1, \ldots, m .
\end{aligned}
$$


We suppose

$$
\bar{f}_{i}(t, x(t) ; u), \bar{g}_{i}(t, x(t), u) \in W^{2, \infty}(Q) .
$$

Under appropriate regularity assumptions on $\partial Q$ and the coefficients, we define and prove existence and uniqueness results of the viscosity solutions to (8).

3.1. Viscosity Solution Definition. It is well known that (8) does not in general have classical smooth solutions. We define a generalized concept of solution called a viscosity solution [7].

Definition $1\left(w \in\left(C_{b}^{2}(Q)\right)^{m}\right)$. $w$ is a viscosity subsolution (supersolution) of system (8), if, for all $\phi_{i} \in C^{2}(\mathcal{Q})$,

$$
\begin{aligned}
& \partial_{t} \phi_{i}\left(t_{0}, x_{0}\right) \\
& \quad+\inf _{u \geq 0}\left\{\frac{1}{2} \bar{g}_{i}\left(t_{0}, x_{0}, u\right) \bar{g}_{i}\left(t_{0}, x_{0}, u\right)^{\prime} D_{x}^{2} \phi_{i}\left(t_{0}, x_{0}\right)\right. \\
& \left.\quad+\bar{f}_{i}\left(t_{0}, x_{0}, u\right) D_{x} \phi_{i}\left(t_{0}, x_{0}\right)\right\}-\sum_{j \neq i} q_{i j}\left[w_{i}\left(t_{0}, x_{0}\right)\right. \\
& \left.\quad-w_{j}\left(t_{0}, x_{0}\right)\right] \geq 0, \\
& \quad+\inf _{u \geq 0}\left\{\frac{1}{2} \bar{g}_{i}\left(t_{0}, x_{0}, u\right) \bar{g}_{i}\left(t_{0}, x_{0}, u\right)^{\prime} D_{x}^{2} \phi_{i}\left(t_{0}, x_{0}\right)\right. \\
& \left.\quad+\bar{f}_{i}\left(t_{0}, x_{0}, u\right) D_{x} \phi_{i}\left(t_{0}, x_{0}\right)\right\}-\sum_{j \neq i} q_{i j}\left[w_{i}\left(t_{0}, x_{0}\right)\right. \\
& \left.\quad-w_{j}\left(t_{0}, x_{0}\right)\right] \leq 0,
\end{aligned}
$$

respectively, whenever $w_{i}-\phi_{i}$ has a local maximum (minimum) at $\left(t_{0}, x_{0}\right) \in Q$; $w$ is a viscosity solution if it is both a viscosity subsolution and supersolution.

\subsection{Uniqueness Result. Next, we can let}

(i)

$$
\begin{aligned}
& \mathscr{H}_{i}(t, x, p, A) \\
& =\inf _{u \geq 0}\left\{\frac{1}{2} \bar{g}_{i}(t, x, u) \bar{g}_{i}(t, x, u)^{\prime} A+\bar{f}_{i}(t, x, u) p\right\} \\
& =-\sup _{u \geq 0}\left\{-\frac{1}{2} \bar{g}_{i}(t, x, u) \bar{g}_{i}(t, x, u)^{\prime} A-\bar{f}_{i}(t, x, u) p\right\},
\end{aligned}
$$

and we assume

(ii)
(a) $\left|\left(\bar{f}_{i}\right)_{t}\right|+\left|\left(\bar{f}_{i}\right)_{x}\right| \leq C,\left|\left(\bar{g}_{i}\right)_{t}\right|+\left|\left(\bar{g}_{i}\right)_{x}\right| \leq C$;
(b) $\left|\bar{f}_{i}(t, x, u)\right| \leq C(1+|x|+|u|)$;
(c) $\left|\bar{g}_{i}(t, x, u)\right| \leq C(1+|x|+|u|)$.

Lemma 2 (see [8], let $\mathscr{H}_{i}$ be as in (13)). Assume ((ii)(a)-(c)). Then, there exists a continuous function $\omega:[0, \infty) \rightarrow$ $[0, \infty)$ that satisfies $\omega(0)=0$ such that

$$
\begin{aligned}
& \mathscr{H}_{i}(t, y, \beta(x-y), B)-\mathscr{H}_{i}(t, x, \beta(x-y), A) \\
& \quad \leq \omega\left(\beta|x-y|^{2}+|x-y|\right),
\end{aligned}
$$

for every $(t, x),(t, y) \in \mathbb{Q}, \beta>0$, and symmetric matrices $A$, $B$ satisfying

$$
-3 \beta\left(\begin{array}{cc}
I & 0 \\
0 & I
\end{array}\right) \leq\left(\begin{array}{cc}
A & 0 \\
0 & -B
\end{array}\right) \leq 3 \beta\left(\begin{array}{cc}
I & -I \\
-I & I
\end{array}\right),
$$

where $I$ is the identity matrix with appropriate dimension.

Proposition 3. Suppose assumptions (9) and (10) hold and $w_{k}$ is a viscosity subsolution of (8) and $v_{k}$ is a supersolution of (8).

$$
\begin{aligned}
& \text { If } w_{k}(t, x) \leq v_{k}(t, x) \text { on } \partial \mathcal{Q}, k=1, \ldots, m, \text { then } \\
& w_{k}(t, x) \leq v_{k}(t, x) \text { on } \mathbb{Q}, k=1, \ldots, m .
\end{aligned}
$$

Proof. Suppose that there does not exist an index, $s$ and $(l, z) \in \mathbb{Q}$, such that

$$
\left(w_{s}-v_{s}\right)(l, z)=\max _{x, t, k}\left\{\left(w_{k}-v_{k}\right)(t, x)\right\}>0 .
$$

(i) If $(l, z) \in \partial Q$, we are done.

(ii) Assume $(l, z) \in \mathbb{Q}$; let

$$
\begin{aligned}
\phi_{r}^{\varepsilon}(t, x, y)= & w_{k}(t, x)-v_{k}(t, y)-\frac{1}{\varepsilon^{2}}|x-y|^{2} \\
& -\varepsilon|t-l|^{2} .
\end{aligned}
$$

There exists an index $r$ and $\left(t_{0}, x_{0}, y_{0}\right)=\left(t_{0}^{\varepsilon}, x_{0}^{\varepsilon}, y_{0}^{\varepsilon}\right)$ such that

$$
\phi_{r}^{\varepsilon}\left(t_{0}, x_{0}, y_{0}\right)=\max _{x, t, y, k}\left\{\phi_{k}^{\varepsilon}(t, x, y)\right\}
$$

We now show

$$
w_{r}\left(t_{0}, x_{0}\right)-v_{r}\left(t_{0}, y_{0}\right) \geq 0 \text {. }
$$

But

$$
\phi_{s}^{\varepsilon}(l, z, z)=w_{s}(l, z)-v_{s}(l, z) \leq \phi_{r}^{\varepsilon}\left(t_{0}, x_{0}, y_{0}\right)
$$

implies

$$
\begin{aligned}
& \frac{1}{\varepsilon^{2}}\left|x_{0}-y_{0}\right|^{2}+\varepsilon\left|t_{0}-l\right|^{2} \\
& \quad \leq w_{r}\left(t_{0}, x_{0}\right)-v_{r}\left(t_{0}, y_{0}\right)-\left(w_{s}(l, z)-v_{s}(l, z)\right) .
\end{aligned}
$$

Since $w_{s}(l, z)-v_{s}(l, z)>0$,

$$
\begin{aligned}
0 & \leq \frac{1}{\varepsilon^{2}}\left|x_{0}-y_{0}\right|^{2}+\varepsilon\left|t_{0}-l\right|^{2} \\
& \leq w_{r}\left(t_{0}, x_{0}\right)-v_{r}\left(t_{0}, y_{0}\right)-\left(w_{s}(l, z)-v_{s}(l, z)\right) \\
& \leq w_{r}\left(t_{0}, x_{0}\right)-v_{r}\left(t_{0}, y_{0}\right) .
\end{aligned}
$$


(i) Since $w$ is a viscosity subsolution of (8) and the function

$$
\Psi_{r, 1}^{\varepsilon}:(x, t) \longmapsto \phi_{r}^{\varepsilon}\left(t, x, y_{0}\right)
$$

has a maximum at $\left(t_{0}, x_{0}\right)$, set

$$
\varphi_{r, 1}^{\varepsilon}(t, x)=v_{r}\left(t, y_{0}\right)+\frac{1}{\varepsilon^{2}}\left|x-y_{0}\right|^{2}+\varepsilon|t-l|^{2},
$$

then $w_{r}(t, x)-\varphi_{r, 1}^{\varepsilon}(t, x)$, has a maximum at $\left(t_{0}, x_{0}\right)$, and hence

$$
\begin{aligned}
& \left.\frac{\partial\left(w_{r}-\varphi_{r, 1}^{\varepsilon}\right)}{\partial t}\right|_{\left(t_{0}, x_{0}\right)}=\left.\frac{\partial\left(w_{r}-\varphi_{r, 1}^{\varepsilon}\right)}{\partial x}\right|_{\left(t_{0}, x_{0}\right)}=0 \Longrightarrow \\
& \left.\frac{\partial \varphi_{r, 1}^{\varepsilon}}{\partial t}\right|_{\left(t_{0}, x_{0}\right)}=\left.\frac{\partial w_{r}}{\partial t}\right|_{\left(t_{0}, x_{0}\right)}, \\
& \left.\frac{\partial \varphi_{r, 1}^{\varepsilon}}{\partial x}\right|_{\left(t_{0}, x_{0}\right)}=\left.\frac{\partial w_{r}}{\partial x}\right|_{\left(t_{0}, x_{0}\right)}, \\
& \left.\frac{\partial \Psi_{r, 1}^{\varepsilon}}{\partial x}\right|_{\left(t_{0}, x_{0}\right)}=0 \Longleftrightarrow \\
& \left.\frac{\partial w_{r}}{\partial x}\right|_{\left(t_{0}, x_{0}\right)}-\frac{2}{\varepsilon^{2}}\left(x_{0}-y_{0}\right)=0, \\
& \left.\frac{\partial \Psi_{r, 1}^{\varepsilon}}{\partial t}\right|_{\left(t_{0}, x_{0}\right)}=0 \Longleftrightarrow \\
& \left.\frac{\partial w_{r}}{\partial t}\right|_{\left(t_{0}, x_{0}\right)}-\left.\frac{\partial v_{r}\left(t_{0}, x_{0}\right)}{\partial t}\right|_{\left(t_{0}, x_{0}\right)}-2 \varepsilon\left(t_{0}-l\right)=0,
\end{aligned}
$$

0

$$
\begin{aligned}
\leq & \frac{\partial \varphi_{r, 1}^{\varepsilon}\left(t_{0}, x_{0}\right)}{\partial t} \\
& -\mathscr{H}_{r}\left(t_{0}, x_{0}, \frac{2}{\varepsilon^{2}}\left(x_{0}-y_{0}\right), D_{x}^{2} \varphi_{r, 1}^{\varepsilon}\left(t_{0}, x_{0}\right)\right) \\
& -\sum_{k \neq r} q_{r k}\left[w_{r}\left(t_{0}, x_{0}\right)-w_{k}\left(t_{0}, x_{0}\right)\right] .
\end{aligned}
$$

(ii) Since $v$ is a viscosity supersolution of (8) and the function

$$
\Psi_{r, 2}^{\varepsilon}:(x, t) \longmapsto-\phi_{r}^{\varepsilon}\left(t, x_{0}, y\right)
$$

has a minimum at $\left(t_{0}, y_{0}\right)$, set

$$
\varphi_{r, 2}^{\varepsilon}(t, y)=w_{r}\left(t, x_{0}\right)-\frac{1}{\varepsilon^{2}}\left|x_{0}-y\right|^{2}-\varepsilon|t-l|^{2}
$$

then $\varphi_{r, 2}^{\varepsilon}(t, x)-v_{r}(t, x)$, has a maximum at $\left(t_{0}, x_{0}\right)$, and hence

$$
\begin{aligned}
& \left.\frac{\partial\left(\varphi_{r, 2}^{\varepsilon}-v_{r}\right)}{\partial t}\right|_{\left(t_{0}, y_{0}\right)}=\left.\frac{\partial\left(\varphi_{r, 2}^{\varepsilon}-v_{r}\right)}{\partial x}\right|_{\left(t_{0}, y_{0}\right)}=0 \Longrightarrow \\
& \left.\frac{\partial \varphi_{r, 2} \varepsilon}{\partial t}\right|_{\left(t_{0}, y_{0}\right)}=\left.\frac{\partial v_{r}}{\partial t}\right|_{\left(t_{0}, y_{0}\right)}, \\
& \left.\frac{\partial \varphi_{r, 2}^{\varepsilon}}{\partial x}\right|_{\left(t_{0}, y_{0}\right)}=\left.\frac{\partial v_{r}}{\partial x}\right|_{\left(t_{0}, y_{0}\right)}, \\
& \left.\frac{\partial \Psi_{r, 2}^{\varepsilon}}{\partial y}\right|_{\left(t_{0}, y_{0}\right)}=0 \Longleftrightarrow \\
& +\left.\frac{\partial v_{r}}{\partial y}\right|_{\left(t_{0}, y_{0}\right)}-\frac{2}{\varepsilon^{2}}\left(x_{0}-y_{0}\right)=0, \\
& \left.\frac{\partial \Psi_{r, 2}^{\varepsilon}}{\partial t}\right|_{\left(t_{0}, x_{0}\right)}=0 \Longleftrightarrow \\
& -\left.\frac{\partial w_{r}}{\partial t}\right|_{\left(t_{0}, x_{0}\right)}+\left.\frac{\partial v_{r}\left(t_{0}, x_{0}\right)}{\partial t}\right|_{\left(t_{0}, x_{0}\right)}+2 \varepsilon\left(t_{0}-l\right)=0, \\
& \frac{\partial \varphi_{r, 2}^{\varepsilon}\left(t_{0}, y_{0}\right)}{\partial t} \\
& -\mathscr{H}_{r}\left(t_{0}, x_{0}, \frac{2}{\varepsilon^{2}}\left(x_{0}-y_{0}\right), D_{y}^{2} \varphi_{r, 2}^{\varepsilon}\left(t_{0}, y_{0}\right)\right) \\
& -\sum_{k \neq r} q_{r k}\left[v_{r}\left(t_{0}, y_{0}\right)-v_{k}\left(t_{0}, y_{0}\right)\right] \leq 0 .
\end{aligned}
$$

By combining (26) and (30),

$$
\begin{aligned}
0 \leq & \frac{\partial w_{r}\left(t_{0}, x_{0}\right)}{\partial t}-\frac{\partial v_{r}\left(t_{0}, y_{0}\right)}{\partial t} \\
& +\mathscr{H}_{r}\left(t_{0}, y_{0}, \frac{2}{\varepsilon^{2}}\left(x_{0}-y_{0}\right), D_{y}^{2} \varphi_{r, 2}^{\varepsilon}\left(t_{0}, y_{0}\right)\right) \\
& -\mathscr{H}_{r}\left(t_{0}, x_{0}, \frac{2}{\varepsilon^{2}}\left(x_{0}-y_{0}\right), D_{x}^{2} \varphi_{r, 1}^{\varepsilon}\left(t_{0}, x_{0}\right)\right) \\
& -\sum_{k \neq r} q_{r k}\left[w_{r}\left(t_{0}, x_{0}\right)-w_{k}\left(t_{0}, x_{0}\right)\right] \\
& +\sum_{k \neq r} q_{r k}\left[v_{r}\left(t_{0}, y_{0}\right)-v_{k}\left(t_{0}, y_{0}\right)\right]
\end{aligned}
$$

and by Lemma 2

$$
\begin{aligned}
0 \leq & 2 \varepsilon\left|t_{0}-l\right|+\omega\left(\frac{2}{\varepsilon^{2}}\left|x_{0}-y_{0}\right|^{2}+\left|x_{0}-y_{0}\right|\right) \\
& -\sum_{k \neq r} q_{r k}\left[w_{r}\left(t_{0}, x_{0}\right)-w_{k}\left(t_{0}, x_{0}\right)\right] \\
& +\sum_{k \neq r} q_{r k}\left[v_{r}\left(t_{0}, y_{0}\right)-v_{k}\left(t_{0}, y_{0}\right)\right]
\end{aligned}
$$


we obtain

$$
\begin{aligned}
0 \leq & 2 \varepsilon\left(t_{0}-l\right)+\omega\left(\frac{2}{\varepsilon^{2}}\left|x_{0}-y_{0}\right|^{2}+\left|x_{0}-y_{0}\right|\right) \\
& -\sum_{k \neq r} q_{r k}\left[w_{r}\left(t_{0}, x_{0}\right)-v_{r}\left(t_{0}, y_{0}\right)\right] \\
& +\sum_{k \neq r} q_{r k}\left[w_{k}\left(t_{0}, x_{0}\right)-v_{k}\left(t_{0}, y_{0}\right)\right] .
\end{aligned}
$$

We have $-q_{r k}<0$ and $w_{r}\left(t_{0}, x_{0}\right)-v_{r}\left(t_{0}, y_{0}\right)>0$ (19) and hence

$$
\begin{aligned}
0 \leq & 2 \varepsilon\left(t_{0}-l\right)+\omega\left(\frac{2}{\varepsilon^{2}}\left|x_{0}-y_{0}\right|^{2}+\left|x_{0}-y_{0}\right|\right) \\
& +\sum_{k \neq r} q_{r k}\left[w_{k}\left(t_{0}, x_{0}\right)-v_{k}\left(t_{0}, y_{0}\right)\right] .
\end{aligned}
$$

Since $w_{k}\left(t_{0}, x_{0}\right)-v_{k}\left(t_{0}, y_{0}\right) \leq w_{r}\left(t_{0}, x_{0}\right)-v_{r}\left(t_{0}, y_{0}\right)$ and $\sum_{k \neq r} q_{r k}=-q_{k k}$, we obtain

$$
\begin{aligned}
0 \leq & 2 \varepsilon\left(t_{0}-l\right)+\omega\left(\frac{2}{\varepsilon^{2}}\left|x_{0}-y_{0}\right|^{2}+\left|x_{0}-y_{0}\right|\right) \\
& -q_{k k}\left[w_{r}\left(t_{0}, x_{0}\right)-v_{r}\left(t_{0}, y_{0}\right)\right] .
\end{aligned}
$$

Thus,

$$
\begin{aligned}
w_{s}(l, z)-v_{s}(l, z) \leq \phi_{r}^{\varepsilon}\left(t_{0}, x_{0}, y_{0}\right) \\
\leq w_{r}\left(t_{0}, x_{0}\right)-v_{r}\left(t_{0}, y_{0}\right) \\
\quad \leq \frac{1}{q_{k k}} 2 \varepsilon\left|t_{0}-l\right| \\
\quad+\frac{1}{q_{k k}} \omega\left(\frac{2}{\varepsilon^{2}}\left|x_{0}-y_{0}\right|^{2}+\left|x_{0}-y_{0}\right|\right) .
\end{aligned}
$$

To finish the proof, we need to show

$$
\omega\left(\frac{2}{\varepsilon^{2}}\left|x_{0}-y_{0}\right|^{2}+\left|x_{0}-y_{0}\right|\right) \longrightarrow 0, \quad \text { as } \varepsilon \longrightarrow 0 .
$$

Let

$$
\begin{aligned}
h(q) & =\operatorname{Sup}\left\{\left|v_{r}(t, x)-v_{r}(t, y)\right|: t \in[0, T],(x, y)\right. \\
& \left.\in \bar{Q}|x-y|^{2} \leq q\right\},
\end{aligned}
$$

so that for any $(t, x)$ and $(t, y) \in \bar{Q}$

$$
\left|v_{r}(t, x)-v_{r}(t, y)\right| \leq h\left(|x-y|^{2}\right) .
$$

Since $\left(x_{0}, t_{0}, y_{0}, r\right)$ maximizes $\phi_{r}^{\varepsilon}$ over $\mathbb{Q}$,

$$
\begin{aligned}
& w_{r}\left(t_{0}, x_{0}\right)-v_{r}\left(t_{0}, y_{0}\right)-\frac{1}{\varepsilon^{2}}\left|x_{0}-y_{0}\right|^{2}-\varepsilon\left|t_{0}-l\right|^{2} \\
& \geq w_{r}\left(t_{0}, x_{0}\right)-v_{r}\left(t_{0}, x_{0}\right)-\frac{1}{\varepsilon^{2}}\left|x_{0}-x_{0}\right|^{2} \\
& \quad-\varepsilon\left|t_{0}-l\right|^{2} .
\end{aligned}
$$

We obtain

$$
\begin{aligned}
\frac{1}{\mathcal{\varepsilon}^{2}}\left|x_{0}-y_{0}\right|^{2} & \leq v_{r}\left(t_{0}, x_{0}\right)-v_{r}\left(t_{0}, y_{0}\right) \\
& \leq h\left(\left|x_{0}-y_{0}\right|^{2}\right) .
\end{aligned}
$$

Since $h$ is bounded by some constant $K$, this implies that

$$
\frac{2}{\varepsilon^{2}}\left|x_{0}-y_{0}\right|^{2} \leq K
$$

The definition of $h$ yields

$$
\frac{2}{\varepsilon^{2}}\left|x_{0}-y_{0}\right|^{2}+\left|x_{0}-y_{0}\right| \leq h\left(\frac{K \varepsilon^{2}}{2}\right)+\varepsilon \sqrt{\frac{K}{2}},
$$

and we obtain

$$
\begin{gathered}
\omega\left(\frac{2}{\varepsilon^{2}}\left|x_{0}-y_{0}\right|^{2}+\left|x_{0}-y_{0}\right|\right) \\
\leq \omega\left(h\left(\frac{K \varepsilon^{2}}{2}\right)+\varepsilon \sqrt{\frac{K}{2}}\right),
\end{gathered}
$$

and we obtain

$$
w_{s}(l, z)-v_{s}(l, z) \leq 0 \quad \text { as } \varepsilon \longrightarrow 0,
$$

which is a contradiction to (16).

Corollary 4. The viscosity solution satisfying the boundary condition is unique.

Proof. If $v_{k}^{1}$ and $v_{k}^{2}$ are 2 viscosity solutions such that $v_{k}^{1}=v_{k}^{2}$ on $\partial Q$, then

(i) $v_{k}^{1} \leq v_{k}^{2}$ on $\partial \mathscr{Q} \Rightarrow v_{k}^{1} \leq v_{k}^{2}$ on $\mathbb{Q}$ accordingly (Proposition 3);

(ii) $v_{k}^{1} \geq v_{k}^{2}$ on $\partial \mathscr{Q} \Rightarrow v_{k}^{1} \geq v_{k}^{2}$ on $\mathbb{Q}$ accordingly (Proposition 3).

Hence $v_{k}^{1}=v_{k}^{2}$ on $Q$.

\section{Application in Finance: Continuous-Time Mean-Variance Model without Shorting where the Market Parameters Are Random}

We now briefly recall the results of the continuous-time mean-variance model without shorting [5] and the meanvariance portfolio selection problem in continuous time where the market parameters are random processes [4].

We study the intersection of the both cases $[4,5]$, that is, continuous-time mean-variance model without shorting where the market parameters are random.

Consider a market in which $n+1$ assets are traded continuously on a finite time horizon $[0, T]$. One of the assets is a bank account whose price $P_{0}(t)$ is subject to the stochastic ODE (ordinary differential equation)

$$
\begin{aligned}
d P_{0}(t) & =r(t, \alpha(t)) P_{0}(t) d t, \quad t \in[0, T] \\
P_{0}(0) & =p_{0}>0, \quad t \in[0, T], \alpha(t)=i \in \mathbb{N},
\end{aligned}
$$


where $r(t, i) \geq 0, i=1,2, \ldots, m$, are given as interest rate processes corresponding to different market modes. The other $n$ assets are stocks whose price processes $P_{m}(t) m=$ $1,2, \ldots, n$ satisfy the system of SDE (system of differential equation)

$$
\begin{aligned}
& d P_{m}(t)=P_{m}(t) \\
& \cdot\left\{b_{m}(t, \alpha(t)) d t+\sum_{p=1}^{n} \sigma_{m p}(t, \alpha(t)) d W_{p}(t)\right\}, \\
& \quad t \in[0, T] \\
& P_{m}(0)=p_{m}>0, \quad t \in[0, T], \alpha(t)=i \in \mathbb{N},
\end{aligned}
$$

where for each $i=1,2, \ldots, n b_{m}(t, i)$ is the appreciation rate process and $\sigma_{m}(t, i)=\left(\sigma_{m 1}(t, i), \ldots, \sigma_{m n}(t, i)\right)$ is the volatility or the dispersion rate process of the $m$ th stock, corresponding to $\alpha(t)=i$.

Define the volatility matrix

$$
\sigma(t, i) \equiv\left(\sigma_{m p}(t, i)\right)_{n \times n} \quad \text { for each } i=1, \ldots, n .
$$

We assume

$$
\sigma(t, i) \sigma(t, i)^{\prime} \geq \delta I \quad \forall t \in[0, T], \delta>0
$$

and $r(t, i), b_{m}(t, i), \sigma_{m n}(t, i)$ are measurable and uniformly bounded in $t$.

Denote by $y(t)$ the total wealth of the agent with $y(0)=$ $y_{0}>0$ being his initial wealth; $y(t)$ satisfies

$$
\begin{aligned}
& d y(t)=[r(t, i) y(t)+B(t, i) u(t) \\
& \left.+\sum_{m=1}^{n}\left[b_{m}(t, i)-r(t, i)\right]\right] u_{m}(t) d t \\
& \quad+\sum_{p=1}^{n} \sum_{m=1}^{n} \sigma_{m p}(t, i) u_{m}(t) d W_{p}(t), \quad t \in[s, T] \\
& y(0)=y_{0}>0, \quad \alpha(0)=i_{0}, \text { the initial market mode, }
\end{aligned}
$$

where $u_{m}(t)$ is the total market value of the agent's wealth in the $m$ th asset and $m=0,1, \ldots, n$ at time $t$.

$u(\cdot)=\left(u_{1}(\cdot), \ldots, u_{n}(\cdot)\right)^{\prime}$ is called a portfolio of the agent.

$u_{0}(\cdot)$, the asset in the bank account, is completely specified since $u_{0}(t)=y(t)-\sum_{i=1}^{n} u_{i}(t)$. Thus, in our analysis to follow, only $u(\cdot)$ is considered.

Setting

$$
\begin{aligned}
& B(t, i)=\left(b_{1}(t, i)-r(t, i), \ldots, b_{n}(t, i)-r(t, i)\right), \\
& i \in \mathscr{M},
\end{aligned}
$$

wealth equation (50) satisfies

$$
\begin{aligned}
d y(t)= & {[r(t, i) y(t)+B(t, i) u(t)] d t } \\
& +u(t)^{\prime} \sigma(t, i) d W(t), \quad t \in[s, T] \\
y(0)= & y_{0}>0, \quad \alpha(0)=i_{0} .
\end{aligned}
$$

The objective of the agent is to find an admissible portfolio $u(\cdot) \geq 0$, whose expected terminal wealth is $E_{t y} y(T)=d$ for a given $d \in \mathbb{R}$, so that the risk is measured by the variance of the terminal wealth. Namely, the goal of the agent is to solve the following constrained stochastic optimization problem, parameterized by $d \in \mathbb{R}$ :

$$
\begin{array}{cl}
\text { minimize } & J_{\mathrm{MV}}\left(y_{0}, i_{0}, u(\cdot)\right)=E_{t y}[y(T)-d]^{2}, \\
\text { subject to } & E_{t y} y(T)=d, \\
& (y(\cdot), u(\cdot)) \text { admissible, }
\end{array}
$$

called mean-variance portfolio.

Formula (53) is a convex optimization problem; by using a Lagrange multiplier $\mu \in \mathbb{R}$, we can attach the equality constraint $E_{t y} y(T)=d$ to the first equation of (53). In this way, the portfolio problem can be solved via the following optimal stochastic control problem:

$P(d)$ :

$$
\begin{aligned}
& \text { minimize } E_{t y}\left\{[y(T)-d]^{2}+2 \mu\left[E_{t y} y(T)-d\right]\right\}, \\
& \text { subject to } E_{t y} y(T)=d
\end{aligned}
$$

$$
(y(\cdot), u(\cdot)) \text { admissible, }
$$

where factor 2 in front of the multiplier $\mu$ is introduced in the objective function just for convenience.

This problem is equivalent to the following:

$(A(\mu))$ :

$$
\begin{array}{cl}
\text { minimize } & E_{t y}\left[\frac{1}{2}[y(T)-(d-\mu)]^{2}\right], \\
\text { subject to } & u(\cdot) \in L_{\mathscr{F}}^{2}\left(0, T ; \mathbb{R}_{+}^{m}\right) \\
& (y(\cdot), u(\cdot)) \text { admissible, }
\end{array}
$$

in the sense that two problems have exactly the same optimal control [5].

Next, we let $x(t)=y(t)-(d-\mu)$.

Consider $(A(\mu))$ :

$$
\begin{array}{cl}
\text { minimize } & E_{t x}\left[\frac{1}{2}[x(T)]^{2}\right], \\
\text { subject to } & u(\cdot) \in L_{\mathscr{F}}^{2}\left(0, T ; \mathbb{R}_{+}^{m}\right) \\
& (x(\cdot), u(\cdot)) \text { admissible, }
\end{array}
$$

and (52) is equivalent to

$$
\begin{aligned}
& d x(t) \\
& =[A(t, i) x(t)+B(t, i) u(t)+A(t, i)(d-\mu)] d t \\
& +\sum_{p=1}^{n} G_{p}(t, i) u(t) d W_{p}(t), \quad t \in[s, T] \\
& x(s)=y(s)-(d-\mu) \in \mathbb{R} \\
& \quad \text { where } G_{p}(t, i)=\left(\sigma_{1 p}(t, i), \ldots, \sigma_{n p}(t, i)\right)
\end{aligned}
$$

$A(t, i) \in \mathbb{R}$. 
Problem $A(\mu)$ is a stochastic optimal linear quadratic coupled (LQC) control problem, and we can get the solution of $(A(\mu))$ by guessing the solution as a quadratic function. By making use of the duality relationship between $(P(d))$ and $(A(\mu))$, see Appendix A.2; we obtain the solution of the original problem $(P(d))$.

4.1. A General Constrained Stochastic Linear Quadratic Problem. Consider controlled linear stochastic differential equation (57).

We assume that the matrix $\sum_{p=1}^{n} G_{p}(t, i)^{\prime} G_{p}(t, i)$ is nonsingular. Our objective is to find an optimal control $u(\cdot)$ that minimizes the quadratic terminal cost function. Set

$$
\mathcal{U}[s, T]=L_{\mathscr{F}}^{2}\left(s, T ; \mathbb{R}_{+}^{m}\right) .
$$

Given $u(\cdot) \in \mathcal{U}[s, T]$, the pair $(x(\cdot), u(\cdot))$ is admissible if $x(\cdot) \in$ $L_{\mathscr{F}}^{2}(s, T ; \mathbb{R})$ is a solution of $(57)$. Let

$$
J_{i}(s, x ; u(\cdot))=E_{t x}\left\{\frac{1}{2} x(T)^{2}\right\} .
$$

The value function associated with LQC problem (57) and (59) is defined by

$$
V_{i}(s, x)=\inf _{u(\cdot) \in \mathscr{U}[s, T]} J_{i}(s, x ; u(\cdot)) .
$$

In Appendix A.3, and also [8], value function (60) satisfies (8). Next, we will provide an explicit viscosity solution of (8).

\section{Definition 5.}

(i) A portfolio $u(\cdot)$ is said to be admissible if $u(\cdot) \epsilon$ $L_{\mathscr{F}}^{2}\left(0, T ; \mathbb{R}_{+}^{n}\right)$ and the SDE (57) has a unique solution $x(\cdot)$ corresponding to $u(\cdot)$. In this case, we refer to $(x(\cdot), u(\cdot))$ as an admissible (wealth, portfolio) pair.

(ii) The problem is called feasible if there is at least one portfolio satisfying all the constraints.

(iii) The problem is called finite if it is feasible and the infimum of $J_{\mathrm{MV}}\left(x_{0}, i_{0}, u(\cdot)\right)$ is finite.

(iv) An optimal portfolio to the above problem, if it ever exists, is called an efficient portfolio corresponding to $d$, and the corresponding $(\operatorname{Var} x(T), d) \in \mathbb{R}^{2}$

and $\left.\left(\sigma_{x(T)}, d\right) \in \mathbb{R}^{2}\right)$ are interchangeably called an efficient point, and the set of all the efficient points is called the efficient frontier.

Next, we let

$$
\begin{aligned}
& \bar{f}_{i}(t, x ; u)=A(t, i) x(t)+B(t, i) u+f(t, i), \\
& \bar{g}_{i}(t, x ; u)=G(t, i) u,
\end{aligned}
$$

$$
\text { where } f(t, i)=A(t, i)(d-\mu) \text {. }
$$

4.2. Viscosity Solution of the Coupled System. By guessing the value function of (8) as

$$
V_{i}(t, x)=\frac{1}{2} P(t, i) x^{2}+M(t, i) x+R(t, i)
$$

we will see that the coefficients of (8) satisfy the following Riccati equation.

Definition 6. We define the system of Riccati equations as follows

$$
\begin{aligned}
& \frac{d \widehat{P}(t, i)}{d t} \\
& =\left[-2 A(t, i)-\|\bar{\xi}(t, i)\|^{2}-2 B_{i}(t) G^{-1}(t, i) \bar{\xi}(t, i)\right] \\
& \cdot \widehat{P}(t, i)+\sum_{j \neq i}^{n} q_{i, j}[\widehat{P}(t, i)-\widehat{P}(t, j)], \\
& \widehat{P}(T, k)=1, \\
& \frac{d \widehat{M}(t, i)}{d t} \\
& \quad=\left[-A(t, i)-\|\bar{\xi}(t, i)\|^{2}-2 B(t, i) G^{-1}(t, i) \bar{\xi}(t, i)\right] \\
& \quad \widehat{M}(t, i)-\widehat{P}(t, i) f(t, i) \\
& +\sum_{j \neq i}^{n} q_{i, j}[\widehat{M}(t, i)-\widehat{M}(t, j)],
\end{aligned}
$$

$\widehat{M}(T, k)=0$,

$$
\begin{aligned}
& \frac{d \widehat{R}(t, i)}{d t}=-\widehat{M}(t, i) f(t, i)-B(t, i) G^{-1}(t, i) \bar{\xi}(t, i) \\
& \cdot \widehat{M}^{2}(t, i) \widehat{P}(t, i)^{-1}-\frac{1}{2}\|\bar{\xi}(t, i)\|^{2} \widehat{M}^{2}(t, i) \widehat{P}(t, i)^{-1} \\
& \quad+\sum_{j \neq i}^{n} q_{i, j}[\widehat{R}(t, i)-\widehat{R}(t, j)]
\end{aligned}
$$

$\widehat{R}(T, k)=0$,

$$
\begin{aligned}
& \frac{d \widetilde{P}(t, i)}{d t}=-2 A(t, i) \widetilde{P}(t, i) \\
& \quad+\sum_{j \neq i}^{n} q_{i, j}[\widehat{P}(t, i)-\widehat{P}(t, j)], \\
& \widehat{P}(T, k)=1, \\
& \frac{d \widetilde{M}(t, i)}{d t}=-A(t, i) \widetilde{M}(t, i)+\widetilde{P}(t, i) f(t, i) \\
& \quad+\sum_{j \neq i}^{n} q_{i, j}[\widetilde{M}(t, i)-\widetilde{M}(t, j)], \\
& \widehat{M}(T, k)=0,
\end{aligned}
$$




$$
\begin{aligned}
& \frac{d \widetilde{R}(t, i)}{d t}=-\widetilde{M}(t, i) f(t, i) \\
& \quad+\sum_{j \neq i}^{n} q_{i, j}[\widetilde{R}(t, i)-\widetilde{R}(t, j)], \\
& \widehat{R}(T, k)=0,
\end{aligned}
$$

where $\bar{\xi}(t, i)$ is as in Lemma A.1

Remark 7. By letting

$$
\begin{aligned}
& \widetilde{\alpha}(t, i)=-2 A(t, i)-\|\bar{\xi}(t, i)\|^{2} \\
&-2 B(t, i) G^{-1}(t, i) \bar{\xi}(t, i), \\
& \tilde{\beta}(t, i)=- 2 A(t, i)-\|\bar{\xi}(t, i)\|^{2} \\
&-2 B(t, i) G^{-1}(t, i) \bar{\xi}(t, i), \\
& \tilde{\gamma}(t, i)=-\widehat{M}(t, i) f(t, i) \\
&-B(t, i) G^{-1}(t, i) \bar{\xi}(t, i) \widehat{M}^{2}(t, i) \widehat{P}(t, i) \\
&-\frac{1}{2}\|\bar{\xi}(t, i)\|^{2} \widehat{M}^{2}(t, i) \widehat{P}(t, i) .
\end{aligned}
$$

We see that (63) is equivalent to

$$
\begin{aligned}
\frac{d \widehat{P}(t)}{d t} & =M(t) \widehat{P}(t), \\
M(t) & =\left[\widetilde{\alpha}(t, i) \delta_{i j}+q_{i j}\right]_{1 \leq i, j \leq n}, \\
\widehat{P}(T) & =1,
\end{aligned}
$$

where $\widehat{P}(t)=(\widehat{P}(t, i))_{1 \leq i \leq m}$;

(64) is equivalent to

$$
\begin{aligned}
\frac{d \widehat{M}(t)}{d t} & =N(t) \widehat{M}(t)+G(t), \\
N(t) & =\left[\widetilde{\beta}(t, i) \delta_{i j}+q_{i j}\right]_{1 \leq i, j \leq n}, \\
G(t) & =\left(\widehat{P}_{1} f_{1}(t) \cdots \widehat{P}_{n} f_{n}(t)\right), \\
\widehat{M}(T) & =0,
\end{aligned}
$$$$
\text { where } \widehat{M}(t)=(\widehat{M}(t, i))_{1 \leq i \leq m} \text {; }
$$

(65) is equivalent to

$$
\begin{aligned}
\frac{d \widehat{R}(t)}{d t} & =Q(t) \widehat{R}(t)+\widetilde{\gamma}(t), \\
Q(t) & =\left[q_{i j}\right]_{1 \leq i, j \leq n}, \\
\widehat{R}(T) & =0,
\end{aligned}
$$

$$
\text { where } \widehat{R}(t)=(\widehat{R}(t, i))_{1 \leq i \leq m} \text {; }
$$

(66) is equivalent to

$$
\begin{aligned}
\frac{d \widetilde{P}(t)}{d t} & =H(t) \widetilde{P}(t), \\
H(t) & =\left[-2 A_{i}(t) \delta_{i j}+q_{i j}\right]_{1 \leq i, j \leq n}, \\
\widetilde{P}(T) & =0,
\end{aligned}
$$

$$
\text { where } \widetilde{P}(t)=(\widetilde{P}(t, i))_{1 \leq i \leq m} \text {; }
$$

(67) is equivalent to

$$
\begin{aligned}
\frac{d \widetilde{M}(t)}{d t} & =L(t) \widetilde{M}(t)+K(t), \\
L(t) & =\left[-A_{i}(t) \delta_{i j}+q_{i j}\right]_{1 \leq i, j \leq n}, \\
K(t) & =\left(\widetilde{P}_{1} f_{1}(t) \cdots \widetilde{P}_{n} f_{n}(t)\right), \\
\widetilde{P}(T) & =0,
\end{aligned}
$$

$$
\text { where } \widetilde{M}(t)=(\widetilde{M}(t, i))_{1 \leq i \leq m} \text {; }
$$

(68) is equivalent to

$$
\begin{aligned}
\frac{d \widetilde{R}(t)}{d t} & =Q(t) \widetilde{R}(t)+O(t), \\
Q(t) & =\left[q_{i j}\right]_{1 \leq i, j \leq n}, \\
O(t) & =\left(\widetilde{M}_{1} f_{1}(t) \cdots \widetilde{M}_{n} f_{n}(t)\right), \\
\widetilde{R}(t) & =0,
\end{aligned}
$$

$$
\text { where } \widetilde{R}(t)=(\widetilde{R}(t, i))_{1 \leq i \leq m} \text {. }
$$

4.3. Riccati Equation Magnus Approach. We will show how to provide the solutions of (70)-(75) by making use of Magnus method.

Proposition 8 (see [10]). Given the $n \times n$ coefficient matrix $A(t)$,

$$
\begin{aligned}
\frac{d Y(t)}{d t} & =A(t) Y(t), \\
Y\left(t_{0}\right) & =Y_{0}
\end{aligned}
$$

$$
\text { where } Y(t)=(Y(t, i))_{1 \leq i \leq m}
$$

and then $Y(t)=\exp \left(\left(\Omega\left(t, t_{0}\right)\right) Y_{0}\right)$ which is subsequently constructed as a series expansion

$$
\begin{aligned}
\Omega\left(t, t_{0}\right) & =\sum_{k=1}^{\infty} \Omega_{k}\left(t, t_{0}\right) \quad \text { where } \Omega_{1}=\int_{t_{0}}^{t} A(\tau) d \tau \\
\Omega_{n}\left(t, t_{0}\right) & =\sum_{j=1}^{n-1} \frac{B_{j}}{j !} \int_{t_{0}}^{t} S_{n}^{(j)}(\tau) d \tau, \quad n \geq 2,
\end{aligned}
$$


where $S_{n}^{j}$ is defined recursively by

$$
\begin{aligned}
S_{n}^{(j)} & =\sum_{m=1}^{n-j}\left[\Omega_{m}, S_{n-m}^{(j-1)}\right], \quad 2 \leq j \leq n-1, \\
S_{n}^{(1)} & =\left[\Omega_{n-1}, A\right], \\
S_{n}^{(n-1)} & =a d_{\Omega_{1}}^{n-1}(A),
\end{aligned}
$$

$a d_{\Omega}^{k}$ iterated commutator

$$
\begin{aligned}
a d_{\Omega} A & =[\Omega, A], \\
a d_{\Omega}^{k+1} A & =\left[\Omega, a d_{\Omega}^{k} A\right], \\
a d_{\Omega}^{0} A & =A,
\end{aligned}
$$$$
k \in \mathbb{N}
$$

and $B_{j}$ is the Bernoulli numbers.

Proposition 9 (see [11]).

$$
\begin{aligned}
& \frac{d Y(t)}{d t}=M(t) Y(t)+Y(t) N(t)+F(t), \\
& Y\left(t_{0}\right)=Y_{0}
\end{aligned}
$$

$$
t \in\left[t_{0}, T\right]
$$

where $Y(t), F(t) \in \mathbb{C}^{p \times q}, M(t) \in \mathbb{C}^{p \times p}$, and $N(t) \in \mathbb{C}^{q \times q}$. The solution of $(80)$ is given by

$$
Y(t)=\Phi_{M}\left(t, t_{0}\right) Y_{0} \Phi_{N}^{*}\left(t, t_{0}\right)+\Psi\left(t, t_{0}\right)
$$

with

$$
\Psi\left(t, t_{0}\right)=\int_{t_{0}}^{t} \Psi_{M}(t, s) F(s) \Phi_{N}^{*}(t, s) d s,
$$

where $\Phi_{m}\left(t, t_{0}\right)$ and $\Phi_{N}^{*}\left(t, t_{0}\right)$ are the fundamental solution matrices of the associated homogeneous equations

$$
\begin{aligned}
\Phi_{M}^{\prime}\left(t, t_{0}\right) & =M(t) \Phi_{M}\left(t, t_{0}\right), \\
\Phi_{M}\left(t_{0}, t_{0}\right) & =I_{p}, \\
\Phi^{* \prime}\left(t, t_{0}\right) & =\Phi^{*}\left(t, t_{0}\right) N(t), \\
\Phi_{N}\left(t_{0}, t_{0}\right) & =I_{q} .
\end{aligned}
$$

Remark 10. By making use of Proposition 8 we get (70) and (71)-(75) are special case of Proposition 9 when $N=\left[0_{i j}\right]_{n \times n}$.

Theorem 11. The value function of $(60)$ is given by

$$
V_{i}(t, x)= \begin{cases}\bar{V}_{i}(t, x)=\frac{1}{2} \widehat{P}(t, i) x^{2}+\widehat{M}(t, i) x+\widehat{R}(t, i), & \text { if } x+\bar{\eta}(t, i) \leq 0, \\ \widetilde{V}_{i}(t, x)=\frac{1}{2} \widetilde{P}(t, i) x^{2}+\widetilde{M}(t, i) x+\widetilde{R}(t, i), & \text { if } x+\bar{\eta}(t, i)>0\end{cases}
$$

and the optimal control is given by

$$
\begin{aligned}
& u^{*} \\
& = \begin{cases}-\left(G(t, i)^{\prime}\right)^{-1} \bar{\xi}(t, i)[x+\bar{\eta}(t, i)], & \text { if } x+\bar{\eta}(t, i) \leq 0, \\
0, & \text { if } x+\bar{\eta}(t, i)>0,\end{cases}
\end{aligned}
$$

where

$$
\bar{\eta}(t, i)=\frac{\widehat{M}(t, i)}{\widehat{R}(t, i)} .
$$

Proof. Let

$$
\begin{aligned}
& \Gamma_{1}^{i}=\{(t, x, i) \in[0, T] \times \mathbb{R} \times \mathbb{N} \mid x+\bar{\eta}(t, i) \leq 0\}, \\
& \Gamma_{2}^{i}=\{(t, x, i) \in[0, T] \times \mathbb{R} \mid x+\bar{\eta}(t, i)>0\} .
\end{aligned}
$$

(i) In $\Gamma_{1}^{i}, V$ as given by (62) is well defined, with

$$
\begin{aligned}
\frac{\partial V_{i}(t, x)}{\partial t} & =\frac{1}{2} \dot{\widehat{P}}(t, i) x^{2}+\dot{\widehat{M}}(t, i) x+\dot{\widehat{R}}(t, i) \\
\frac{\partial V_{i}(t, x)}{\partial x} & =\widehat{P}(t, i) x+\widehat{M}(t, i), \\
\frac{\partial^{2} V_{i}(t, x)}{\partial x^{2}} & =\widehat{P}(t, i) .
\end{aligned}
$$

Substituting them into the left-hand side (LHS) of (8), we obtain

$$
\begin{aligned}
\text { LHS } & =\left(\frac{1}{2} \dot{\hat{P}}(t, i)+\widehat{P}(t, i) A(t)+\frac{1}{2}\right. \\
& \left.\cdot \sum_{j \neq i} q_{i j}(\widehat{P}(t, i)-\widehat{P}(t, j))\right) x^{2}(\dot{\widehat{M}}(t, i)+\widehat{P}(t, i) \\
& \cdot f(t, i)+\widehat{M}(t, i) A_{i}(t)
\end{aligned}
$$




$$
\begin{aligned}
& \left.+\sum_{j \neq i} q_{i j}(\widehat{R}(t, i)-\widehat{R}(t, i))\right) x+(\dot{\hat{R}}(t, i)+\widehat{M}(t, i) \\
& \left.\cdot f(t, i)+\sum_{j \neq i} q_{i j}(\widehat{R}(t, i)-\widehat{R}(t, i))\right) \\
& \cdot \inf _{u \geq 0}\left[\frac{1}{2} u^{\prime} G(t, i)^{\prime} G(t, i) u\right. \\
& \left.+B(t, i)\left(x+\frac{\widehat{M}(t, i)}{\widehat{P}(t, i)}\right) u\right] .
\end{aligned}
$$

Let $\bar{\eta}(t, i)=\widehat{M}(t, i) / \widehat{P}(t, i)$ and, by using Lemma A.1 $\bar{\alpha}=-[x+\bar{\eta}(t, i)]>0$, it follows that the minimizer of (89) is achieved by

$$
u^{*}=-\left(G(t, i)^{\prime}\right)^{-1} \bar{\xi}(t, i)[x+\bar{\eta}(t, i)] .
$$

Substituting $u^{*}(t, x)$ back into (8) and noting (63)(65), it immediately follows that LHS $=0$.

Now, we will show that $\bar{V}$ is a viscosity subsolution.

Let $\varphi_{i} \in C^{2}(\mathcal{Q})$ and choose $(\bar{t}, \bar{x}) \in \operatorname{argmax}\left\{\left(\bar{V}_{i}-\right.\right.$ $\left.\left.\varphi_{i}\right)(t, x) \mid(t, x) \in \bar{Q}\right\} \cap \mathbb{Q}$; then,

$$
\begin{aligned}
\frac{\partial \varphi_{i}(\bar{t}, \bar{x})}{\partial t} & =\frac{\partial \bar{V}_{i}(\bar{t}, \bar{x})}{\partial t}, \\
\frac{\partial \varphi_{i}(\bar{t}, \bar{x})}{\partial x} & =\frac{\partial \bar{V}_{i}(\bar{t}, \bar{x})}{\partial x}, \\
\frac{\partial^{2}\left(\bar{V}_{i}-\varphi_{i}\right)}{\partial x^{2}}(\bar{t}, \bar{x}) & \leq 0 \Longrightarrow \\
\bar{P}_{i} & =\frac{\partial^{2} \bar{V}_{i}(\bar{t}, \bar{x})}{\partial x^{2}} \leq \frac{\partial^{2} \varphi_{i}(\bar{t}, \bar{x})}{\partial x^{2}}
\end{aligned}
$$

and we obtain

$$
\begin{aligned}
0= & \partial_{t} \bar{V}_{i}(\bar{t}, \bar{x}) \\
& +\inf _{u \geq 0}\left\{\frac{1}{2} \bar{g}_{i}(\bar{t}, \bar{x}, u) \bar{g}_{i}(\bar{t}, \bar{x}, u)^{\prime} D_{x}^{2} \bar{V}_{i}(\bar{t}, \bar{x})\right. \\
& \left.+\bar{f}_{i}(\bar{t}, \bar{x}, u) D_{x} \bar{V}_{i}(\bar{t}, \bar{x})\right\}-\sum_{j \neq i} q_{i j}\left[\bar{V}_{i}(\bar{t}, \bar{x})\right. \\
& \left.-\bar{V}_{j}(\bar{t}, \bar{x})\right] \leq \partial_{t} \varphi_{i}(\bar{t}, \bar{x}) \\
& +\inf _{u \geq 0}\left\{\frac{1}{2} \bar{g}_{i}(\bar{t}, \bar{x}, u) \bar{g}_{i}(\bar{t}, \bar{x}, u)^{\prime} D_{x}^{2} \varphi_{i}(\bar{t}, \bar{x})\right. \\
& \left.+\bar{f}_{i}(\bar{t}, \bar{x}, u) \varphi_{i}(\bar{t}, \bar{x})\right\}-\sum_{j \neq i} q_{i j}\left[\bar{V}_{i}(\bar{t}, \bar{x})\right. \\
& \left.-\bar{V}_{j}(\bar{t}, \bar{x})\right] .
\end{aligned}
$$

Hence, $\bar{V}_{i}$ is a viscosity subsolution. (ii) In $\Gamma_{2}^{i}$, we proceed similarly with

$$
\begin{aligned}
\frac{\partial V_{i}(t, x)}{\partial t} & =\frac{1}{2} \dot{\widetilde{P}}(t, i) x^{2}+\dot{\widetilde{M}}(t, i) x+\dot{\widetilde{R}}(t, i) \\
\frac{\partial V_{i}(t, x)}{\partial x} & =\widetilde{P}(t, i) x+\widetilde{M}(t, i), \\
\frac{\partial^{2} V_{i}(t, x)}{\partial x^{2}} & =\widetilde{P}(t, i) .
\end{aligned}
$$

Substituting them into the left-hand side (LHS) of (8), we obtain

$$
\begin{aligned}
& \text { LHS }=\left(\frac{1}{2} \dot{\widetilde{P}}(t, i)+\dot{\widetilde{P}}(t, i) A(t, i)+\frac{1}{2}\right. \\
& \left.\cdot \sum_{j \neq i} q_{i j}(\dot{\widetilde{P}}(t, i)-\dot{\widetilde{P}}(t, j))\right) x^{2}(\dot{\tilde{M}}(t, i)+\widetilde{P}(t, i) \\
& \cdot f_{i}(t)+\widetilde{M}(t, i) A(t, i) \\
& \left.+\sum_{j \neq i} q_{i j}(\widetilde{R}(t, i)-\widetilde{R}(t, j))\right) x+(\dot{\widetilde{R}}(t, i)+\dot{\widetilde{M}}(t, i) \\
& \left.\cdot f(t, i)+\sum_{j \neq i} q_{i j}(\widetilde{R}(t, i)-\widetilde{R}(t, j))\right) \\
& +\inf _{u \geq 0}\left[\frac{1}{2} u^{\prime} G(t, i)^{\prime} G_{i}(t) u\right. \\
& \left.+B(t, i)\left(x+\frac{\widetilde{M}(t, i)}{\widetilde{P}(t, i)}\right) u\right] .
\end{aligned}
$$

Since $\alpha=-[x+\bar{\eta}(t, i)]>0$, the minimizer of (94) is

$$
u_{i}^{*}=0
$$

Substituting $u^{*}$ into (8), it is easy to show that $\widetilde{V}$ satisfies HJBC equation (8) in $\Gamma_{2}^{i}$.

Now, we will show that $\widetilde{V}$ is a viscosity subsolution.

Let $\phi_{i} \in C^{2}(\widetilde{Q})$ and choose $(\tilde{t}, \tilde{x}) \in \operatorname{argmin}\left\{\left(\widetilde{V}_{i}-\right.\right.$ $\left.\left.\varphi_{i}\right)(t, x) \mid(t, x) \in \bar{Q}\right\} \cap \mathbb{Q}$; then,

$$
\begin{aligned}
& \frac{\partial \phi_{i}(\tilde{t}, \tilde{x})}{\partial t}=\frac{\partial \widetilde{V}_{i}(\tilde{t}, \tilde{x})}{\partial t}, \\
& \frac{\partial \phi_{i}(\tilde{t}, \tilde{x})}{\partial x}=\frac{\partial \widetilde{V}_{i}(\widetilde{t}, \tilde{x})}{\partial x},
\end{aligned}
$$




$$
\begin{aligned}
\frac{\partial^{2}\left(\widetilde{V}_{i}-\phi_{i}\right)}{\partial x^{2}}(\widetilde{t}, \tilde{x}) & \geq 0 \Longrightarrow \\
\widetilde{P}_{i} & =\frac{\partial^{2} \widetilde{V}_{i}(\widetilde{t}, \tilde{x})}{\partial x^{2}} \geq \frac{\partial^{2} \phi_{i}(\widetilde{t}, \tilde{x})}{\partial x^{2}}
\end{aligned}
$$

and we obtain

$$
\begin{aligned}
0= & \partial_{t} \widetilde{V}_{i}(\widetilde{t}, \tilde{x}) \\
& +\inf _{u \geq 0}\left\{\frac{1}{2} \bar{g}_{i}(\widetilde{t}, \widetilde{x}, u) \bar{g}_{i}(\widetilde{t}, \widetilde{x}, u)^{\prime} D_{x}^{2} \widetilde{V}_{i}(\widetilde{t}, \widetilde{x})\right. \\
& \left.+\bar{f}_{i}(\widetilde{t}, \widetilde{x}, u) D_{x} \widetilde{V}_{i}(\widetilde{t}, \widetilde{x})\right\}-\sum_{j \neq i} q_{i j}\left[\widetilde{V}_{i}(\widetilde{t}, \tilde{x})\right. \\
& \left.-\widetilde{V}_{j}(\widetilde{t}, \tilde{x})\right] \geq \partial_{t} \phi_{i}(\widetilde{t}, \tilde{x}) \\
& +\inf _{u \geq 0}\left\{\frac{1}{2} \bar{g}_{i}(\widetilde{t}, \widetilde{x}, u) \bar{g}_{i}(\widetilde{t}, \widetilde{x}, u)^{\prime} D_{x}^{2} \phi_{i}(\widetilde{t}, \tilde{x})\right. \\
& \left.+\bar{f}_{i}(\widetilde{t}, \tilde{x}, u) \phi_{i}(\widetilde{t}, \tilde{x})\right\}-\sum_{j \neq i} q_{i j}\left[\widetilde{V}_{i}(\widetilde{t}, \tilde{x})\right. \\
& \left.-\widetilde{V}_{j}(\widetilde{t}, \widetilde{x})\right] .
\end{aligned}
$$

Hence, $\widetilde{V}_{i}$ is a viscosity supersolution.

We see that the value function $V$ is a viscosity solution.

Remark 12 . we see clearly that $\partial^{2} V(t, x, i) / \partial x^{2}$ does not exist in $\mathcal{Q}$, since $\widetilde{P}(t, i) \neq \widehat{P}(t, i)$. For this reason, we are required to work within the framework of viscosity solutions.

\section{Efficient Strategies}

Consider $x(t)=y(t)-(d-\mu)$. The problem $A(\mu)$ is equivalent to the following problem:

$$
\begin{aligned}
\min \quad E_{t x} & {\left[\frac{1}{2} x(T)\right] } \\
d x(t) & \\
= & {[A(t, i) x(t)+B(t, i) u+f(t, i)] d t } \\
& +G(t, i) u d W(t), \quad t \in[s, T] \\
x & (0)=y_{0}-(d-\mu),
\end{aligned}
$$

where $u(\cdot) \in L_{\mathscr{F}}^{2}\left(0, T ; \mathbb{R}_{+}^{m}\right)$ and

$$
\begin{aligned}
A(t, i) & =r(t, i), \\
B(t, i) & =\left(b_{1}(t, i)-r(t, i), \ldots, b_{n}(t, i)-r(t, i)\right), \\
f(t, i) & =(d-\mu) r(t, i), \\
G(t, i) & =\left(\sigma_{i 1}(t, i), \ldots, \sigma_{i n}(t, i)\right) .
\end{aligned}
$$

Now, corresponding to (A.3), set

$$
\begin{aligned}
& \bar{\pi}_{i}(t)=\underset{\pi(t, i) \in[0, \infty)^{m}}{\operatorname{argmin}} \frac{1}{2} \| \sigma(t, i)^{-1} \pi(t, i) \\
& \quad+\sigma(t, i)^{-1}(b(t, i)-r(t, i) \mathbf{1}) \|^{2}, \\
& \bar{\theta}_{i}(t)=\sigma_{i}(t)^{-1} \pi(t, i)+\sigma_{i}(t)^{-1}(b(t, i)-r(t, i) \mathbf{1}) .
\end{aligned}
$$

5.1. An Optimal Strategy. We present the optimal investment strategy for the problem $A(\mu)$. The optimal control obtained in (85) translates into the following strategy:

$$
\begin{aligned}
& u^{*} \equiv\left(u_{1}^{*}, \ldots, u_{m}^{*}\right)^{\prime}, \\
& u^{*}= \begin{cases}-\left(\sigma(t, i)^{\prime}\right)^{-1} \bar{\theta}(t, i)[y+(d-\mu)+\bar{\eta}(t, i)], & \text { if } y+(d-\mu)+\bar{\eta}(t, i) \leq 0, \\
0, & \text { if } y+(d-\mu)+\bar{\eta}(t, i)>0 .\end{cases}
\end{aligned}
$$

Theorem 13. The optimal investment strategy to the problem $A(\mu)$ is given by (101).

\section{Efficient Frontier}

Since $x(t)=y(t)-(d-\mu)$, we obtain the solution of the original problem $P(D)$. Hence, for every fixed $\mu$, we have

$$
\begin{gathered}
\min _{u(\cdot) \in \mathscr{U}[0, T]} \quad E_{t y}\left\{\frac{1}{2}[y(T)-d]^{2}\right\}+\mu\left[E_{t y} y(T)-d\right] \\
=\min _{u(\cdot) \in \mathscr{U}[0, T]} E_{t x}\left\{\frac{1}{2} x(T)^{2}\right\}-\frac{1}{2} \mu^{2}
\end{gathered}
$$

$$
=V_{i_{0}}(0, x)-\frac{1}{2} \mu^{2} .
$$

Hence, the value function of $P(D)$ is given:

$$
\begin{gathered}
V_{i_{0}}(0, x)-\frac{1}{2} \mu^{2}=\left(\frac{1}{2} \widehat{P}\left(0, i_{0}\right)\left[y_{0}-(d-\mu)\right]^{2}\right. \\
\left.+\widehat{M}\left(0, i_{0}\right)\left[y_{0}-(d-\mu)\right]+\widehat{R}\left(0, i_{0}\right)-\frac{1}{2} \mu^{2}\right) \\
\cdot 1_{y+(d-\mu)+\bar{\eta}(t, i) \leq 0}+\left(\frac{1}{2} \widetilde{P}\left(0, i_{0}\right)\left[y_{0}-(d-\mu)\right]^{2}\right.
\end{gathered}
$$




$$
\begin{aligned}
& \left.+\widetilde{M}\left(0, i_{0}\right)\left[y_{0}-(d-\mu)\right]+\widetilde{R}\left(0, i_{0}\right)-\frac{1}{2} \mu^{2}\right) \\
& \cdot 1_{y+(d-\mu)+\bar{\eta}(t, i)>0} .
\end{aligned}
$$

Note that the above value still depends on the Lagrange multiplier $\mu$. To obtain the optimal value function, one needs to maximize the value of $\mu$ in (103).

Proposition 14. The efficient strategy of portfolio selection problem (50) corresponding to the expected terminal wealth $E_{t y} y(T)=d$, as a function of time $t$ and wealth $y$, is

$$
u^{*}= \begin{cases}-\left(\sigma(t, i)^{\prime}\right)^{-1} \bar{\theta}(t, i)\left[y+\left(d-\mu^{*}\right)+\eta(t, i)\right], & \text { if } y+\left(d-\mu^{*}\right)+\eta(t, i) \leq 0, \\ 0, & \text { if } y+\left(d-\mu^{*}\right)+\eta(t, i)>0 .\end{cases}
$$

Moreover if

$$
\begin{aligned}
\mu^{*} & =\underset{\mu}{\operatorname{argmax}}\left(\left(\frac{1}{2} \widehat{P}\left(0, i_{0}\right)\left[y_{0}-(d-\mu)\right]^{2}\right.\right. \\
& \left.+\widehat{M}\left(0, i_{0}\right)\left[y_{0}-(d-\mu)\right]+\widehat{R}\left(0, i_{0}\right)-\frac{1}{2} \mu^{2}\right) \\
& \cdot 1_{y+(d-\mu)+\bar{\eta}(t, i) \leq 0}+\left(\frac{1}{2} \widetilde{P}\left(0, i_{0}\right)\left[y_{0}-(d-\mu)\right]^{2}\right. \\
& \left.+\widetilde{M}\left(0, i_{0}\right)\left[y_{0}-(d-\mu)\right]+\widetilde{R}\left(0, i_{0}\right)-\frac{1}{2} \mu^{2}\right) \\
& \left.\cdot 1_{y+(d-\mu)+\bar{\eta}(t, i)>0}\right)
\end{aligned}
$$

exists, the efficient frontier is given by

$$
\operatorname{Var} y(T)=V\left(0, x, i_{0}\right)-\frac{1}{2} \mu^{* 2} \text {. }
$$

\section{Concluding Remarks}

We analyzed mean-variance optimal portfolio selection for a market with regime switching. The formulation allows the market to have random switching with no-shorting constraint. Using techniques of stochastic linear quadratic control and the notion of viscosity solution, mean-variance efficient portfolio and efficient frontiers are derived explicitly in closed forms in terms of some systems of Riccati equation for which the solutions are provided by making use of the Magnus approach. The numerical application is in progress and it will be the subject of a new research paper.

\section{Appendix}

\section{A. Useful Formulas}

\section{A.1. Convex Analysis}

Lemma A.1 (see [5]). Let $h$ be a continuous, strictly convex quadratic function

$$
\begin{aligned}
h(z(t, i))= & \frac{1}{2} z(t, i)^{\prime} \mathscr{D}(t, i)^{\prime} \mathscr{D}(t, i) z(t, i) \\
& -\alpha \mathscr{B} z(t, i)
\end{aligned}
$$

over $z(t, i) \in[0, \infty)^{m}$, where $\mathscr{B}^{\prime} \in \mathbb{R}_{+}^{m}, \mathscr{D}_{i} \in \mathbb{R}^{m \times m}$ and $\mathscr{D}^{\prime}(t, i) \mathscr{D}(t, i)>0$.

For every $\alpha \geq 0, h$ has the unique minimizer $\alpha \mathscr{D}(t, i)^{-1} \bar{\xi}(t, i) \in[0, \infty)^{m}$, where

$$
\bar{\xi}(t, i)=\left(\mathscr{D}(t, i)^{\prime}\right)^{-1} \bar{z}(t, i)+\left(\mathscr{D}(t, i)^{\prime}\right)^{-1} \mathscr{B}^{\prime},
$$

where

$$
\begin{gathered}
\bar{z}(t, i)=\underset{z(t, i) \in[0, \infty)^{m}}{\operatorname{argmin}} \frac{1}{2} \|\left((\mathscr{D}(t, i))^{\prime}\right)^{-1} z(t, i) \\
+\left((\mathscr{D}(t, i))^{\prime}\right)^{-1} B(t, i)^{\prime} \| .
\end{gathered}
$$

\section{A.2. Duality Method}

Lemma A.2 (see [12]). The strong duality relationship holds between $(P(d))$ and $(\mathscr{A}(\mu))$ in the following sense,

$$
\mathscr{V}(P(d))=\max _{\mu \in \mathbb{R}}\left\{2 \mathscr{V}(\mathscr{A}(\mu))-\mu^{2}\right\}
$$

where $\mathscr{V}(\cdot)$ denotes the optimal value of problem $(\cdot)$.

A.3. Dynamic Programming and Random Evolution with Markov Chain Parameters. Here we sketch a proof of equation (8); for more details please see [8].

Let $\alpha(t)$ be a finite state Markov chain, with state space a finite set $\mathscr{M}$. we regard $\alpha(t)$ as a parameter process. On any interval where $\alpha(t)=\alpha$ is constant, $x(t)$ satisfies the ordinary differential equation

$$
\begin{aligned}
d x= & \mu(t, x(t), u(t), \alpha(t)) d t \\
& +\sigma(t, x(t), u(t), \alpha(t)) d w(t)
\end{aligned}
$$

and we assume that $\mu(t, x(t), u(t), \alpha(t))$ and $\sigma(t, x(t), u(t)$, $\alpha(t))$ satisfy the conditions
(i) $\left|\mu_{t}(t, x(t), u(t), \alpha(t))\right|+\left|\mu_{x}(t, x(t), \alpha(t))\right| \leq C$, $\left|\sigma_{t}(t, x(t), u(t), \alpha(t))\right|+\left|\sigma_{x}(t, x(t), u(t), \alpha(t))\right| \leq C ;$
(ii) $|\mu(t, x(t), u(t), \alpha(t))| \leq C(1+|x|+|u|)$;
(iii) $|\sigma(t, x, u(t), \alpha(t))| \leq C(1+|x|+|u|)$, 
for each $\alpha \in \mathscr{M}$. Let $s \leq t \leq T$, and let $\tau_{1}<\tau_{2}<\cdots<\tau_{m}$ denote the successive jump times of the parameter process $\alpha(t)$ during $[s, T]$. We let $\tau_{0}=t, \tau_{m+1}=T$, and define $x(t)$ by

$$
\begin{aligned}
d x= & \mu\left(t, x(t), u(t), \alpha\left(\tau_{i}^{+}\right)\right) d t \\
& +\sigma\left(t, x(t), u(t), \alpha\left(\tau_{i+1}\right)\right) d w(t) \\
& \tau_{i} \leq t<\tau_{i+1}, i=0, \ldots, m, \ldots x(s)=x,
\end{aligned}
$$

with the requirement that $x(\cdot)$ is continuous at each jump time $\tau_{i}$. The process $x(s)$ is not Markov. However, $(x(t), \alpha(t))$ is a Markov process, with state space $\Sigma=\mathbb{R} \times \mathscr{M}$. For each $\Phi(t, x(t), \alpha(t))$ such that $\Phi(\cdot, \cdot, \alpha) \in C^{2}(\bar{Q})$, we have

$$
\begin{aligned}
A^{u} \Phi(t, x, i) \\
=\lim _{x \rightarrow 0} h^{-1}\left[E_{t x} \Phi(t+h, x(t+h), i)-\Phi(t, x, i)\right] \\
=\Phi_{t}(t, x, i)+\frac{1}{2} \sigma(t, x, u, i) D_{x}^{2} \Phi(t, x, i) \\
\quad+\mu(t, x, u, i) D_{x} \Phi(t, x, i) \\
\quad+\sum_{j \neq i} \rho(t, i, j)[\Phi(t, x, j)-\Phi(t, x, i)] .
\end{aligned}
$$

Dynkin formula is

$$
\begin{aligned}
E_{t x} & \Phi(t, x, i)-\Phi(t, x, i) \\
& =E_{t x} \int_{t}^{t_{1}} A^{u(s)} \Phi(s, x(s), \alpha(s)) d s,
\end{aligned}
$$

where $\rho(t, x, y)$ represents an infinitesimal rate at which $x(t)$ jumps from $x$ to $y$ :

$$
\begin{aligned}
\rho(t, i, j) & =\lim _{h \rightarrow 0} h^{-1} P[x(t+h)=j \mid x(t)=i] \\
& =q_{i j}(t) .
\end{aligned}
$$

Criterion to Be Optimized. The control problem of a finite time interval $t \leq s \leq T$ is to minimize

$$
J=E_{t x}\left\{\int_{t}^{T} L(s, x(s), u(s)) d s+\psi x(T)\right\}
$$

in our case the Lagrangian $L(t, x, u) \equiv 0$, that is, the Mayer form.

The value function

$$
V_{i}(t, x)=\inf _{C} J(t, x, i \text {; control }) .
$$

Bellman's Principe of Dynamic Programming. This states that for $t \leq t+h \leq T$

$$
V_{i}(t, x)=\inf _{C} E_{t x} V(t+h, x(t+h), i) .
$$

If we take constant control $u(s)=v$ for $t \leq s \leq t+h$,

$$
V_{i}(t, x) \leq E_{t x} V(t+h, x(t+h), i)
$$

we substract $V(t, x, i)$ from both sides, divided by $h$, and let $h \rightarrow 0$ :

$$
\begin{aligned}
& \lim _{x \rightarrow 0^{+}} h^{-1}\left[\mathbb{E}_{t x} V(t+h, x(t+h), i)-V(t, x, i)\right] \\
& =\lim _{x \rightarrow 0^{+}} h^{-1} \mathbb{E}_{t x} \int_{t}^{t+h} A^{v} V(s, x(s), i) d s \\
& =A^{v} V(t, x, i) .
\end{aligned}
$$

Hence, for all $v \in U$,

$$
0 \leq A^{v} V(t, x, i) .
$$

On the other hand, if $\underline{u}^{*}$ is an optimal Markov control policy, we should have

$$
V_{i}(t, x)=E_{t x} V\left(t+h, x^{*}(t+h), i\right),
$$

where $x^{*}(s)$ is the Markov process generated by $A^{\underline{u}^{*}}$. A similar argument gives, under sufficiently strong assumption (including continuity of $\underline{u}^{*}$ at $(t, x)$ ),

$$
0=A^{\underline{u}^{*}} V(t, x, i) .
$$

Inequatlities (A.15) and (A.17) are equivalent to the dynamic programming equation

$$
0=\min _{v \in U} A^{v} V(t, x, i) .
$$

\section{Competing Interests}

The author declares that no competing interests exist.

\section{References}

[1] H. M. Markowitz, "Portfolio selection," The Journal of Finance, vol. 7, no. 1, pp. 77-91, 1952.

[2] D. Li and W.-L. Ng, "Optimal dynamic portfolio selection: multi-period mean variance formulation," Mathematical Finance, vol. 10, no. 3, pp. 387-406, 2000.

[3] X. Y. Zhou and D. Li, "Continuous-time mean-variance portfolio selection: a stochastic LQ framework," Applied Mathematics and Optimization, vol. 42, no. 1, pp. 19-33, 2000.

[4] X. Y. Zhou and G. Yin, "Markowitz's mean-variance portfolio selection with regime switching: a continuous-time model," SIAM Journal on Control and Optimization, vol. 42, no. 4, pp. 1466-1482, 2003.

[5] X. Li, X. Y. Zhou, and A. E. Lim, "Dynamic mean-variance portfolio selection with no-shorting constraints," SIAM Journal on Control and Optimization, vol. 40, no. 5, pp. 1540-1555, 2002.

[6] G.-L. Xu and S. E. Shreve, "A duality method for optimal consumption and investment under short-selling prohibition. I. General market coefficients," The Annals of Applied Probability, vol. 2, no. 1, pp. 87-112, 1992.

[7] M. G. Crandall and P.-L. Lions, "Viscosity solutions of Hamilton-Jacobi equations," Transactions of the American Mathematical Society, vol. 277, no. 1, pp. 1-42, 1983.

[8] W. H. Fleming and H. M. Soner, Controlled Markov Processes and Viscosity, Springer, 2nd edition, 2006. 
[9] S. M. Lenhart, "Viscosity solutions for weakly coupled systems of first-order partial differential equations," Journal of Mathematical Analysis and Applications, vol. 131, no. 1, pp. 180-193, 1988.

[10] S. Blanes, F. Casas, J. A. Oteo, and J. Ros, "The Magnus expansion and some of its applications," Physics Reports, vol. 470, no. 5-6, pp. 151-238, 2009.

[11] S. Blanes and E. Ponsoda, "Time-averaging and exponential integrators for non-homogeneous linear IVPs and BVPs," Applied Numerical Mathematics, vol. 62, no. 8, pp. 875-894, 2012.

[12] R. C. Merton, "An analytical derivation of the efficient portfolio frontier," The Journal of Financial and Quantitative Analysis, vol. 7, no. 4, pp. 1851-1872, 1972. 


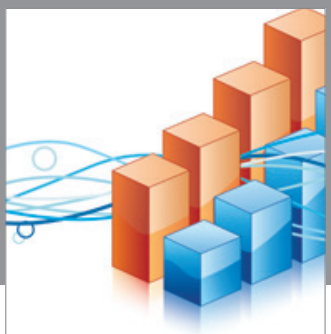

Advances in

Operations Research

vatem alat4

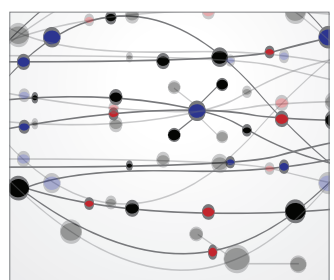

\section{The Scientific} World Journal
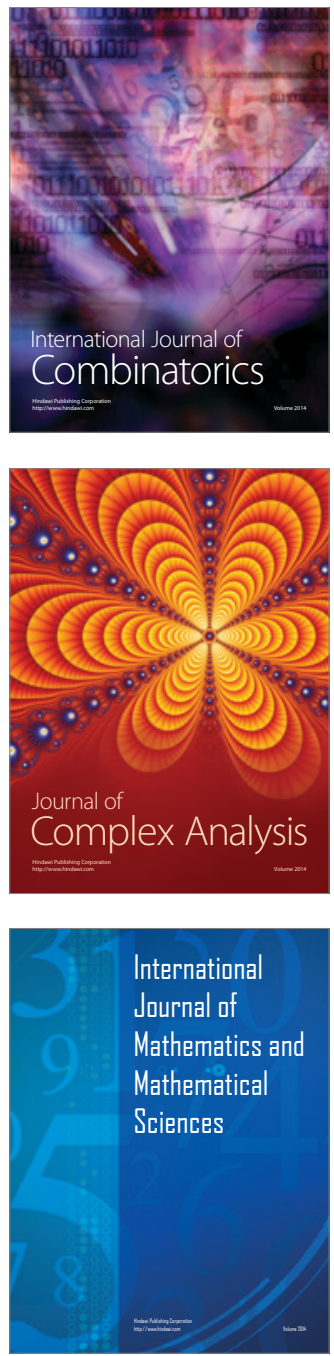
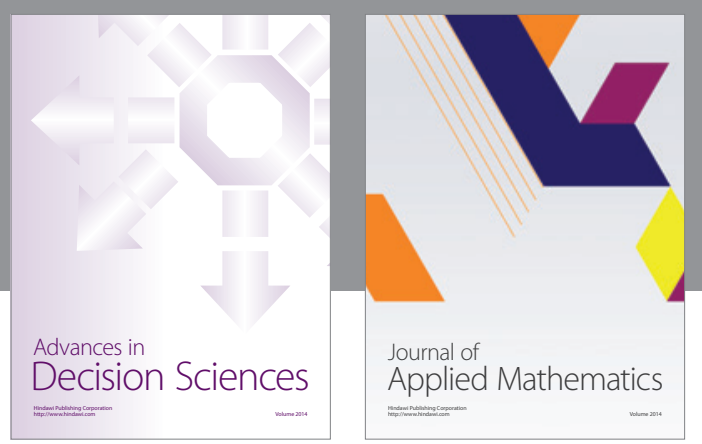

Algebra

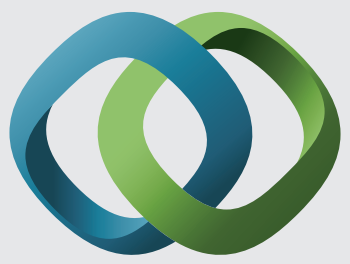

\section{Hindawi}

Submit your manuscripts at

http://www.hindawi.com
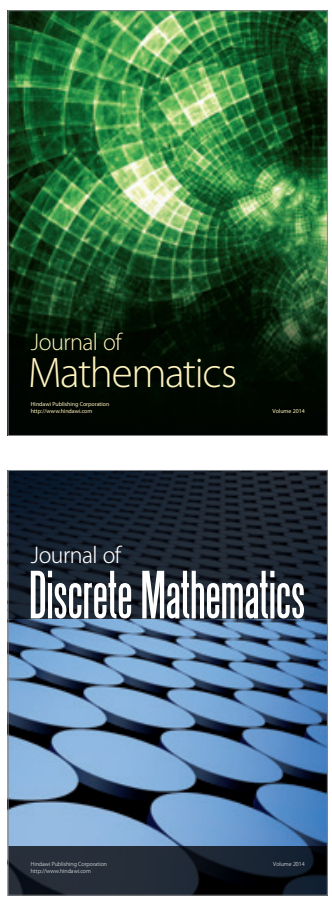

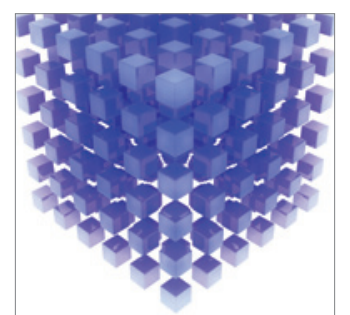

Mathematical Problems in Engineering
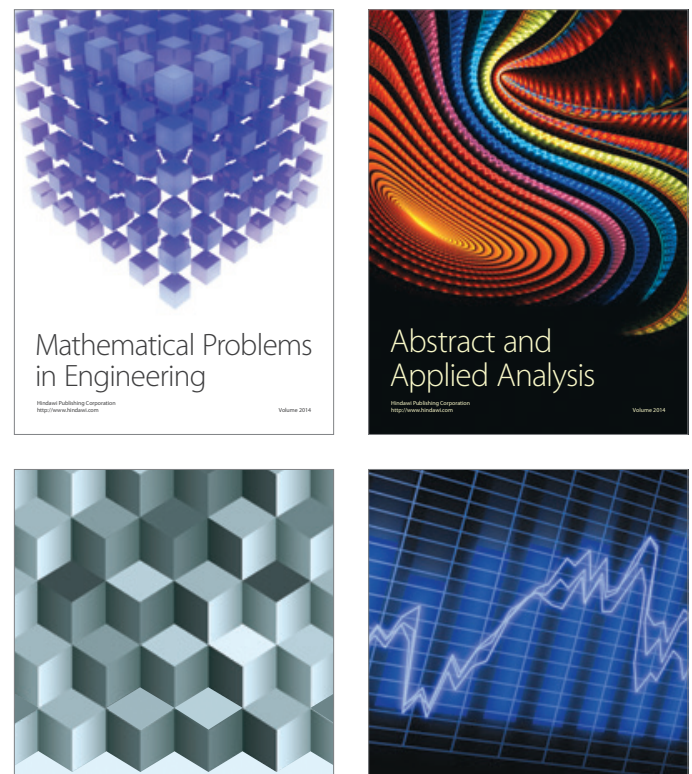

Journal of

Function Spaces

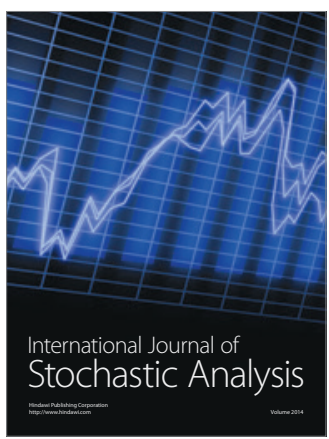

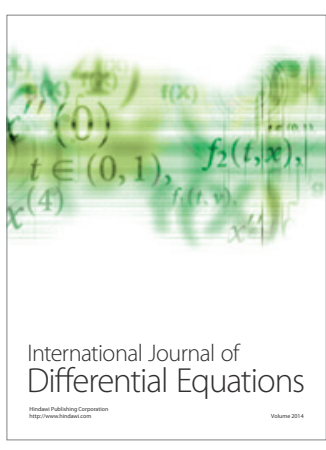
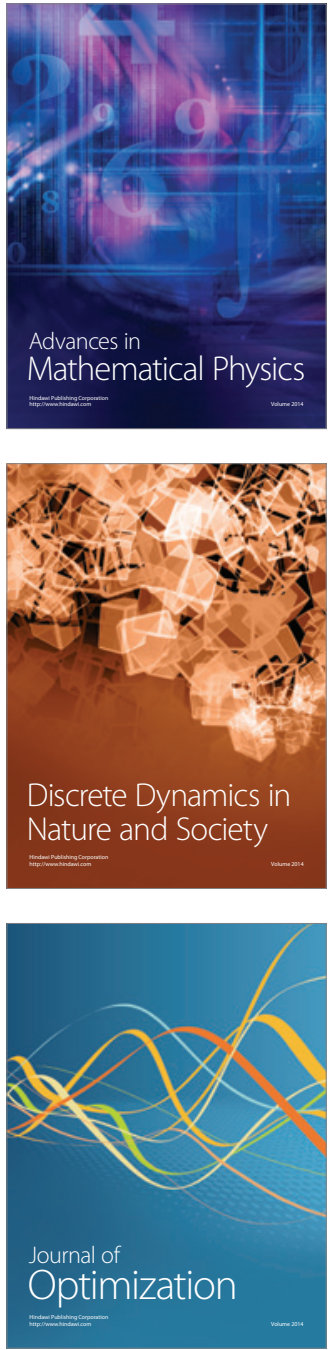